\title{
Le Mariage Et La Dot Dans Les Guvres De Buchi Emecheta Et De Calixthe Beyala
}

\author{
O.F. Siwoku-Awi \\ http://dx.doi./org/10.4314/ujah.v20i2.8
}

\section{Résumé}

Le contexte réaliste qui inspire les æuvres des deux romancières va influencer cette étude des personnages féminins créés par Buchi Emecheta et Calixthe Beyala qui seront analysés dans leur contexte romanesque et seront reliés à la réalité quotidienne. Les deux auteures sont ressortissantes des cultures et des aires linguistiques différentes: Buchi Emecheta, anglophone du Nigeria et Calixthe Beyala, francophone du Cameroun. Bien que l'on soutienne l'idée que les Africains partagent les memes cultures, cet article vise à concrétiser cette opinion tout en relevant également les convergences et les divergences dans les perspectives des deux auteures. Les personnages ont été etudiés dans le cadre patriarcal qui occasionne leur réaction et détermine leur disposition psychique. Cet article mettra en relief quelques exigences patriarcales contraignantes et aliénantes qui relèguent la femme au deuxième rang et étouffent son existence. Il abordera le sujet du mariage et de la dot qui sont les moyens adoptés par le système patriarcat pour contrôler le corps et la sexualité de la femme. Les auteures amènent aussi le lecteur à se retrouver dans le fil de narration des évènements réels auxquels il pourrait s'identifier.

Mots clés: sexualité, dot, mariage, contraintes patriarcales 


\section{Introduction}

La sexualité se présente comme un problème qui suscite des discussions à n'en pas finir partout dans le monde. Les œuvres de nos deux auteures, aussi bien que des reportages actuels fassent référence au jour le jour aux femmes violées, battues et violentées pour des raisons de sexualité. À la une des actualités, est le cas de nombreuses femmes qui ont été tuées en raison d'honneur au Pakistan. Le 19 Novembre 2014, la Radio France Internationale a fait le reportage de plus de huit cents femmes qui ont été tuées au Pakistan pour leur refus de se conformer aux exigences patriarcales de l'Islam, leur religion. L'éducation occidentale et formelle a exercé une influence énorme et des changements indéterminés dans le cadre de rapports homme/femme. Néanmoins, les exigences sexistes et des préjugés contre la femme restent incontournables et indomptables. Sauf, dans le très peu nombre de cultures matrilinéaires, la plupart des cultures patriarcales de l'Afrique accordent l'hégémonie aux hommes si bien qu'ils aient la raison aux cas où ils sont coupables de la violation des droits des femmes.

Dans quelques histoires analysées dans cet article, l'objectif est faire voir que la civilisation est loin d'achever l'émancipation de la femme des traditions contraignantes qui les empêchent de s'épanouir ou vivre aussi longtemps que la nature aurait préféré. En témoignent sont les exemples des filles forcées à se marier et à procréer à l'âge tendre lorsqu'elles n'atteignent pas la maturation physiologique propice à l'enfantement. Ce sujet reste pertinent à la vie actuelle. En adoptant la socio-critique, cette étude fait une explication de certains romans de Beyala et d'Emecheta à l'égard de la dot et le mariage.

Selon Hélène Sabbah, il faut aussi tenir compte de l'environnement socio-cuturel qui détermine la rédaction des textes 
et c'est dans ce cadre que les tendances féministes des auteures sont considérées indissociables des thèmes qu'elles ont choisis. Surtout qu'au départ l'objectif des auteures aurait été de lutter pour faire valoriser la femme africaine. Force est de signaler que Calixthe Beyala et Buchi Emecheta concrétisent par leurs récits la théorie de Molara Ogundipe-Leslie qui propose le Stiwanisme, une variante du féminisme africain comme solution aux problèmes de l'Africaine, empruntant à l'idée du leader chinois, Mao-Tsö-Tong. L'Africaine a sur son dos des montagnes à s'en débarasser et l'une d'elles est constituée des traditions héritées imposées par nos aïeux et qui l'empêchent de grandir. Ce sont quelques unes des traditions héritées qui font l'objet de cette discussion. Sont-elles complètement éradiquées? Faut-il supposer que nos sociétés africaines ne soient plus en danger par la pratique continue de ces traditions? Ne s'exercent-elles pas des effets néfastes sur la psychologie collective et en particulier sur la psyché de la femme africaine ? La fillette jouirait-t-elle un avenir sans préjugé au sein de la famille et dans la société, ou devrait-elle se conformer infinement aux exigences de la procréation, du mariage et du patriarcat? Ne faut-il pas des remues-ménages et des pourparlers pour lancer un combat continu contre les crimes familiaux autour du mariage que l'on appelle des traditions, les traditions qui immobilisent la jeune fille et la femme et les restreignent au niveau des marchandises à manipuler pour se satisfaire ? Le taux des femmes qui meurent de la torture pour leur refus de se compromettre augmente alors que l'homme africain s'accroche au pouvoir. Si les femmes souffrent en permanence des préjugés et de la violence comment pourraient-elles léguer un esprit sain aux enfants ? Ne sont-ils pas les séquelles de la mauvaise enfance non surveillée dont nous souffrons en Afrique au nom de l'insurgence? La femme serait-elle à la hauteur de sa performance au foyer, au 
travail et dans la société si elle n'est pas en bonne santé mentale ? Par ailleurs, ce sont ces traditions qui lui sont imposées qui constituent les entraves à son épanouissement. Il va de soi que nos problèmes sociaux et familiaux aient des rapports directs avec le foyer. Le berceau fournit la stabilité mentale et les atouts pour l'adaptation dans une société en mutation. Dans cet article le sujet porte sur le mariage et la dot et leurs effets sur la femme et en fin de compte le désir de l'homme qui s'attache à cette pratique.

\section{Le mariage et la dot}

Dans certaines cultures africaines il est remarqué qu'avant l'introduction de la culture et la loi occidentale une femme n'avait pas de droit de choisir son mari. Selon Simone de Beauvoir, il était également le cas en Europe traditionnelle et patriarcale que ce fût le père qui fit le choix du mari à sa fille et se mit d'accord avec le soupirant sur la dot à lui remettre en tant que beau-fils. En Afrique c'était toute une autre tradition. C'était le mariage arrangé qui réservait au père de faire le choix pour sa fille et très souvent le gagnant était le plus riche. Dans la pratique patriarcale le plus offrant devenait le mari. La dot payée aux parents pour approprier la fille était comme une somme pour l'acheter et par conséquent elle perdait sa liberté et elle pouvait être traitée comme une esclave. Emecheta fait référence à cette tradition dans la plupart de ses romans. Dans son livre The Slave Girl, la fille protagoniste Aku-nna porte le nom qui fait l'écho de ce que son père attend qu'il soit payé une grande dot par son mari. Le nom signifie la richesse du père. À la mort de son père, son oncle, Okonkwo, veut donc jouir de cette richesse et il se nourrit d'idée que quelqu'un payera une somme considérable surtout que la fille est instruite. Il espère utiliser l'argent pour prendre un titre traditionnel. 
Cet événement va causer la mort d'Akunna qui refuse carrément d'épouser l'homme préféré par son oncle. Elle est amoureuse de Chike, un fils d'esclave qui est son enseignant à l'école. Elle prend la fuite avec celui-ci, elle devient enceinte et souffre moralement et psychologiquement que sa dot n'a pas été payée. Malheureusement son oncle qui renonce à son mariage parce que Chike, son mari est fils d'un ancien esclave lui jète un mauvais sort et fabrique une poupée pour représenter son tchi et la manipule et lui parle de loin à travers la statuette. Selon l'auteure, Aku-nna ne serait pas morte de la malédiction encourrue par le fait que la dot n'est pas payée, mais du fait qu'elle se sent coupable. La réalité médicale juge que la fille est anémique, trop mince, malnourrie et trop petite de taille pour avoir un bébé à l'âge de seize ans et selon la médicine occidentale, sa mort est justifiée par le diagnostic de l'anémie. Le sujet de mariage précoce est soulevé et dénoncé par l'auteure en raison médicale. Sans doute beaucoup de crises médicales résultent du tel mariage. Le mariage précoce est une tradition qui subsiste toujours dans certaines régions du Nigéria et de l'Afrique. Les féministes et les Organisations Nongouvernementales luttent contre cette tradition. La dot se paie toujours, en particulier parmi les igbos du Nigeria, et elle s'élève selon le niveau d'instruction scolaire que la femme aurait reçu avant le mariage et sa beauté, alors que dans d'autres tribus comme les yoroubas elle peut être une somme forfaitaire et symbolique représentant un échange d'amitié des deux familles, car le mariage ne se fait pas seulement par le couple mais par deux familles étendues.

Le problème de la dot ne se pose pas dans The Joys of Motherhood, Nnu Ego la fille de Nwokocha Agbadi se marie en grande pompe avec une large dot qu'elle amène chez son mari. Son 
généreux père voulant que sa fille soit confortable lui donne beaucoup de biens.

The people of Ibuza were never to forget the night the people of Umu-Iso came for Nnu Ego. Her father excelled himself. He accepted the normal bride price, to show that he gave his blessing to the marriage.but he sent his daughter away with seven hefty men and seven young girls carrying her personal possessions. There were seven goats, baskets and baskets of yams, yards and yards of white man's cloth, twenty-four home spun lappas, rows and rows of Hausa trinkets and coral beads (30).

Les gens d'Ibuza ne pouvaient jamais oublier la nuit que les gens d'Umu-Iso étaient venus amener Nnu Ego. Son père s'était surpassé. Il avait accepté le taux de dot en cours, pour démontrer qu'il avait approuvé le mariage, aussi il avait donné sa fille en même temps que chargeant sept hommes costaud et sept jeunes femmes de porter ses effets personnels. Il y avait sept chèvres, des paniers et des paniers d'ignames, des mètres et des mètres du pagne de l'homme blanc, vingt-quatre pagnes de fabrication domestique et des rangs de bijoux haoussa et des colliers de corail.

Malheureusement malgré cette générosité Nnu Ego subit un handicap; elle ne devient pas enceinte aussitôt et son mari Amatokwu l'envoit aux champs pour moissonner des ignames, une corvee des esclaves et des femmes de deuxieme rang, alors qu'il prend une nouvelle femme. La nouvelle femme accouche d'un garçon et par conséquent Nnu Ego est privée de la position chérie de la femme aînée. Si l'institution de la dot améliore le statut de la 
femme dans un foyer occidental, c'est toute une autre histoire chez la femme africaine, où l'emphase porte sur ce que l'homme paie en monnaie et en nature pour s'approprier d'une femme. Selon les cultures de certaines sociétés africaines, c'est comme si la femme est "vendue" à un prix fixé par la dot qui compromet sa liberté; elle devient la servante en chef, si elle a des domestiques et elle est privée de ses droits parce que l'homme l'a achetée. Cette idée est projetée dans The Slave Girl, dans lequel la fille Ojebeta est deux fois esclave: vendue par son frère à l'âge de neuf ans et plus tard à l'adolescence elle devient la femme d'un homme qui paie la dot à ses frères et également rembourse la somme payée pour l'acheter.

Calixthe Beyala présente des scènes plus contemporaines. Ses protagonistes sont sous la tutelle des mères qui commercialisent les corps de leurs filles. À la différence, Emecheta présente le contexte traditionnel où la fille doit être vierge pour qualifier à avoir la dot et où le père ou un parent masculin est receveur de la dot. La tante d'Ateba dans C'est le Soleil qui m'a brûlée, insiste que l'enfant soit vierge afin qu'elle puisse en tirer d'une grande dot si la fille se marie. Elle doit soumettre la fille au rite de l'œuf par lequel une vieille femme fouille son vagin pour déterminer qu'elle n'a pas été dépucelée. Cette question est remise sur le tapis dans La négresse rousse où la plupart des filles subissent le rite de l'œuf. Dame Maman, la mère de Mégrita desire avoir un gendre qui pourrait lui payer une grande dot, malgré le fait que la fille soit enceinte par un autre homme. Beyala soulève que la sexualité de la fille est la propriété de sa mère qui détermine qu'elle soit mariée ou prostituée. La mère de Tanga colle un destiné de prostituée à ses filles qui doivent se commercialiser pour nourrir la famille. La différence est remarquée entre les deux œuvres que c'est l'homme qui prend la dot dans la culture représentée par Emecheta alors que c'est la femme qui la reçoit 
dans l'œuvre de Beyala. Les femmes yoroubas animent la célébration du mariage traditionnel et reçoivent la dot alors que parmi les haoussas ce sont les hommes qui décident et dirigent les cérémonies des noces.

\section{L'homme, que vaut-il ?}

Un autre sujet abordé par les auteures est le statut des hommes Africains qui sont présentés commes des vauriens. Francis, un homme quasi moderne, le mari d'Adah dans le Second Class Citizen d'Emecheta insiste que sa femme paie sa scolarité lorsqu'ils arrivent à Londres et elle doit aussi envoyer de l'argent à sa famille au Nigéria. Cependant Adah n'est plus en contact avec son propre frère nommé Boy qui est obligé de se débrouiller seul étant devenu orphelin. Quand Francis est sous la contrainte de prendre un emploi temporaire pendant la période de noël, au bureau de poste de Londres, il passe tout son temps à se plaindre. Malgré ses méconduites il se voit comme le patron. Dans The Slave Girl, Pa Palagada vit aux dépens de sa femme, Ma Palagada, une grande commerçante. La position de chef de famille est automatiquement et naturellement réservée aux hommes et pour avoir un foyer heureux la femme doit se soumettre aux exigences de l'homme. Les types de maris que Buchi Emecheta peints dans ses œuvres ne sont pas conciliants, ils s'imposent tandis qu'ils puent de décadence morale, ils manquent d'initiative et ils sont des fainéants. En outre, leur comportement violent exerce des effets funestes sur l'inconscient de leurs femmes. Certaines femmes deviennent folles suites à des fracas familiaux. La capacité psychologique des femmes de s'affirmer est réduite ayant été forcées de se soumettre et de faire le choix de la docilité.

Beyala peint pareillement des hommes qu'elle réduit à leur sexe. Beyala de sa part n'accorde pas beaucoup d'importance au 
mariage. Les couples dont elle fait la description sont au milieu de désaccord qui menace la stabilité du foyer. M'ammaryam s'engage dans un rapport illicite avec M. Tichit parce que son mari ne l'aime pas autant que celui-ci. Elle commence à voir des étoiles et des fleurs et elle se berçe d'illusions parce que le nouvel amant est de plus grande capabilité sexuelle que son mari, Abdou. Ce qui est commun à tous les maris dans l'œuvre des femmes est l'incapacité des hommes qui ne peuvent pas gagner le pain pour leur famille. Abdou, un personnge dans Maman a un amant compte beaucoup sur le revenu de sa femme M'am, qui travaille dur et peut payer des vacances pour toute la famille. C'est lors des vacances qu'elle rencontre M. Tichit. A partir de son rapport avec M.Tichit M'am apprend que l'épanouissement de la femme ne serait pas limité aux travaux domestiques ou à la maternité. M. Tichit lui dit qu'il est content de l'avoir comme compagne même si elle ne peut pas avoir un bébé. Les femmes traditionnelles ne peuvent pas s'indigner de l'hégémonie de l'homme, puisqu'elles ne sont pas instruites en plus elles ne se sentent pas trompées par les hommes qui s'imposent et qui oblitèrent leurs intérêts par leurs vetos parce qu'elles sont habituees à la culture quoique discriminatoire et méprisante qu'elle soit. La redoutable prédominance est ressentie dans le mariage où l'homme est beaucoup plus âgé que la femme; Francis a dix ans plus agé qu'Adah. Adah est psychologiquement torturée par le fait qu'elle ne peut pas revendiquer son droit.

Il est à noter que l'homme est ravi du fait qu'il a une femme et il peut la manipuler comme une possession personnelle qui flatte sa vanité sociale; pour lui la femme est un objet de se vanter comme une parcelle de terre ou une maison ou une subordonnée qui doit le servir. Ce point de vue change avec le temps mais il doit être signalé que l'homme le plus indigent croit qu'il a un bien parce qu'il a une femme. Le héro de The Taming of 
the Shrew de Shakepeare invite ses voisins de venir voir la manière dont il domine sa femme de façon autoritaire. La même scène se reproduit à nos jours avec les cas de l'agression de la femme pour ses moindres erreurs rapportés dans les quotidiens. On peut affirmer que ces tendances patriarcales ont été oblitérées par la civilisation, mais avec trépidation parce que le taux élevé de violence contre les femmes est une preuve que les hommes sousestiment toujours les femmes. L'homme dans un état plaisant veut avoir la femme pour tout le bien que celle-ci lui apporte; elle chauffe son lit, fait la cuisine, le ménage, le nettoyage, la lessive et le raccommodage de ses habits et elle prend soins de lui lorsqu'il est malade.

À l'encontre d'Emecheta, Beyala ne décrit pas un personnage féminin qui dispose de la capacité et la maturité pour établir et de faire un foyer. Il semble que le roman Les honneurs perdus présente de manière plus détaillée que les autres œuvres la vie en famille. Bénérafa s'affaire de garder sa femme et sa fille sous son autorité. Même lorsque la femme s'échine à nourrir la famille, elle est obligée d'obéir complètement à son mari. De l'autre côté Papa Bon Blanc dans La négresse rousse est rejété par Dame Maman parce qu'il ne peut pas nourrir sa famille ni payer la dot à la famille de la femme. Elle se donne à plusieurs hommes afin d'avoir de l'argent, y compris le chef du village. Selon Beyala le mariage n'a pas de valeur. Ce sont les femmes qui paient dans le mariage où la valeur de l'homme est surestimée. C'est la femme qui se sacrifie pour nourrir l'homme et la famille. La mère de Saïda est résignée et supporte toutes les mainmises de Bénérafa sur la famille. Même si son mari ne gagne pas de l'argent pour l'entretien de la famille, la mère de Saïda lui fait plaisir par élever sa fille selon les codes islamiques, une religion à laquelle Bénérafa 
est soudainement converti à la naissance de Saïda. La mère de Saïda dit à sa fille:

Il ne faut pas regarder les hommes dans les rues, c'est moche...il est conseillé d'attendre le mariage pour s'adonner à certains plaisirs...Dieu a prevu un époux pour chaque femme et, un jour, cela passe comme Dieu l'a prévu...la virginité et la fidélité sont les plus beaux cadeaux qu'une femme puisse faire à son mari...Etre une femme est un lourd fardeau, ma fille (65).

L'image féminine que l'homme préfère est la femme résignée, ménagère, excitante, obéissante et naïve. La femme délicate est beaucoup plus attirante à l'homme comme un objet sexuel à choyer et à chouchouter alors qu'elle est soumise et passive. Elle ne doit pas parler quand l'homme parle. Quelques femmes acceptent cette position de l'oppression et de l'enfermement au nom de l'amour. Ceci est l'oppression psychologique et émotionnelle. La question pertinente à poser est s'il existe la satisfaction dans l'expérience sexuelle quand la femme est battue. L'homme jouit beaucoup plus d'avantages que la femme d'une telle relation amoureuse. Beyala nous dit de la bouche de son personnage, Yvette, l'une des Débrouillardes, du roman Asseze, l'Africaine, à Assèze "C'est l'amour qui pousse les femmes à accepter l'esclavage."

Bertha dans La négresse rousse ne se marie pas avec papa bon Blanc ni avec le Pygmée, ses deux amants, parcequ'elle ne veut pas céder sa liberté. Mégrita dit à propos de sa maman

J'observai cette bouche aséchée qui, durant des années, n'avait pas esquissé à l'une de mes papas le OUI qui aurait apaisé leur éternelle incertitude, signant à travers le mariage le pacte de l'esclavage. Porter l'eau. Cuisiner. 
Repasser. Ouvrir corps au mâle. Donner son ventre à la maternité (83).

De sa part, Beyala présente que le mariage est comme l'esclavage où la vie de la femme tourne autour de l'homme. Dans Maman a un amant, M'am lamente:

Là-bas, dans mon pays, j'ai baissé les yeux devant mon père, comme ma mere avant moi, comme avant elle ma grand-mère. Les hommes ordonnaient:

"Prends-donne-fais". Les femmes obéissaient. Ainsi allait la vie, ainsi continuait-elle.

Là-bas, dans mon pays les femmes ont les yeux si tristes que toutes les sources du Mali paraissent y venir mourir, hors d'espérance (37).

Forcer la femme à accepter la faiblesse comme vertu et la condition pour l'amour serait inacceptable pour la femme moderne. La plupart des femmes instruites préfèrent s'affirmer et revendiquer leur indépendance. C'est une conciliation déplorable et inéquilibrée que la femme doit concéder dans un argument qui pèse contre l'homme. Le rapport marital et la pratique patriarcale sont en faveur de l'homme et ils constituent la tradition désignée pour contrôler la sexualité de la femme et de la lier perpétuellement à l'homme. La position féministe que Beyala favorise est que l'homme tire beaucoup plus d'avantages que la femme qui paie le plus pour propager la tradition ainsi il faudrait l'annihilation totale de la race masculine, en particulier, en Afrique où les hommes se démontrent incapabales de gouverner les pays et sont complètement émasculés par les traditions qui encouragent leur faux image de soi.

En outre, Emecheta ne présente non plus une position assez agréable du mariage. La femme est obligée de se marier, parce que 
la culture exige qu'elle appartienne à un homme. Dans The Slave Girl, Ojebeta dit que chaque femme est une propriété d'un homme soit par l'achat comme esclave soit par le mariage. Dans The Joys of Motherhood, Nnu Ego dit à la cour lors du procès de son mari Nnaife. "Nnaife is the head of our family. He owns me, just like God in the sky owns us. So even though I pay the fees, yet he owns me. So in other words he pays". (Nnaife est le chef de notre famille. Il me possède, tout comme Dieu au ciel nous possède. Quoique je paie les frais de scolarité, il me possède. En d'autres mots il paie.) D'une facon sarcastique, Emecheta dénonce cette existence parasitique de l'homme et que malgré ses défauts il doit être le chef de famille et le plus rapproché à Dieu. Sa position est plus radicale et révolutionnaire dans Double Yoke quand elle présente une perspective moderne du mariage. La femme instruite n'est plus un produit à offirir aux enchères pour l'homme à s'approprier. La femme est un partenaire qui peut revendiquer ses droits, parce qu'elle peut gagner sa vie et maintenir son indépendance.

\section{Conclusion}

L'approche sociocritique est réaliste et elle permet que le lecteur réfléchisse aux rapports continus entre la littérature et la vie contemporaine rend valoir la littérature africaine comme vivante et pertinente pour revendiquer l'amélioration de la vie de l'individu en particulier la femme africaine dont il s'agit dans cette étude. Selon la recherche et l'analyse faites des textes et ce que l'on sait des réalités qui font la une au quotidien, la vie de la femme africaine n'a pas tellement changé, en vu les reportages dans les quotidiens à l'égard du mépris que lui portent des hommes. Actuellement, le sujet recurrent des reportages au Nigeria porte sur l'abduction des écolières par la secte islamique «Boko Haram » 
depuis le mois d'avril de 2014, il y a plus d'un an que le gouvernement nigérian n'a pas pu emporter la victoire sur ce groupe terroriste. Faire une telle infraction au vingt-unième siècle est renverser toute l'idée de la démocratie et lancer une guerre à la quête collective des nations du monde pour la paix. Ce sont les lois religieuses et les cultures traditionnelles qui sont souvent à la base de la dominance de la sexualité féminine. L'imposition d'un époux à la jeune fille/femme doit être annulée. Faut-il accepter une loi qui prescrit qu'une femme musulmane soit tuée parce qu'elle a épousé un Chrétien? Au cours de l'année 2014, la condamnation du couple Américain dans le Soudan a soulevé une opposition très vive de partout dans le monde car la femme a été condamnée à l'exécution pour avoir épousé un Chrétien. Elle devrait être tuée quand elle aurait achevé la période de l'allaitement de son bébé. Personne n'a pensé à ce que deviendrait l'enfant. Souvent l'homme n'est jamais coupable même lorsqu'il est en délit. Chacun a le droit de choisir sa vie, néanmoins les époux doivent être conseillés des conséquences de leur choix lorsqu'ils ne partagent pas les mêmes croyances religieuses. Les expériences appuient que de tels mariages peuvent tourner au mal, lorsque les parents et la société n'acceptent pas l'union et qu'un partenaire refuse de se fléchir aux croyances de l'autre. Souvent c'est la femme qui souffre.

L'histoire nous fait savoir que les outrances des croisades au Moyen Age contrastent à la tolérance de l'Église d'aujourd'hui, ce qui veut dire que chaque religion peut mener un projet de développement spirituel et d'une politique socio-culturelle qui permettraient une cohabitation paisible des gens appartenant aux tendances différentes. Il faut tout simplement respecter le droit du prochain sans les préjugés de race et de croyance. Évidemment, lors des croisades, la sauvagerie inhérente à l'homme avait été à la 
base des tuéries. Or, par la suite, les teneurs de la pureté du Christianisme ont pu répandre la civilisation biblique qui a permis de fonder la démocratie que les Etats-Unis championne. Les idées religieuses arriérées qui manquent de la rationalité doivent être abandonnées au profit de l'amour du prochain sans lui imposer des conditions.

L'éducation de la femme s'avère comme très importante pour qu'elle puisse prendre en charge sa sexualité et par conséquent les aléas de la culture auxquels elle devrait se conformer. De plus, son indépendance économique est assurée par son instruction et son apprentissage formels. Si par consensus les écrivaines africaines n'ont pas jusqu'à ce point pu à elles-mêmes éradiquer les préjugés contre leurs comportements, on ne doit pas fermer l'œil aux actions phénoménales de certains africains et des Organisations non-gouvernementales qui sensibilisent les sociétés à propos des effets néfastes de la discrimination sexuelle. Bien entendu, ce serait irréalisable d'annuler les conditions traditionnelles contraignantes du mariage et de la dot; pourtant ce sont des aspects de notre vie qui affirme notre humanité et l'effort de les soummetre à la raison nous propulse au delà de la primitivité. Donner sa fille comme une marchandise aux enchères au nom de la dot est déplorable ou s'engager à s'entretuer pour avoir une femme (le sanchi chez les peuhls) ou pour faire un enfant ou tuer la femme en raison d'honneur ou parce qu'elle apportera la honte à la famille, car elle ne succombe pas aux traditions méprisantes sont des crimes contre l'humanité. Si nous nous accrochons à nos sentiments de fidélité au patrimoine, le progrès demande qu'on puisse raisonner pour établir si les pratiques d'antan pourraient nous servir à cette époque de vingt-et-unième siècle. Par la pitié et par l'intégrité les souffrances de la femme dite faible pourraient être minimisées par l'intervention de l'homme qui 
se voit le plus fort. Peu importe l'époque, la galanterie ne perdra jamais sa valeur, sa pertinence son honneur et son éloge. Pourtant, selon nos romancières, l'homme africain est trop égoïste qu'il se montre indigner de fléchir aux besoins de la femme.

\author{
O.F. Siwoku-Awi \\ Department of French \\ University of Ilorin, \\ Kwara State \\ awijesusethos@yahoo.com
}




\section{Bibliographie}

Beyala, Calixthe. C'est le soleil qui m'a brûlée. Paris: Editions Stock, 1987.

---. Les honneurs perdus. Paris: Le Pré aux Clercs Éditions Belford, 1990.

---. Maman a un amant. Paris. Éditions Albin Michel S.A. 1993.

--- Asseze, l'Africaine. Paris. Éditions Albin Michel, 1994

--- La Négresse rousse. Paris. Éditions Albin Michel1996.

Borgomano, Madeleine. «Calixthe Beyala, une écriture déplacée. » Notre librairie, No 125 janvier-mars, 1996: 7274.

Borgomano, Madeleine. «Les femmes et l'écriture-parole. » Paris: Notre librairie. No 117, avril-juin 1994 : 87-94.

Cazenave, Odile. « 40 ans d'écriture au féminin. » Notre librairie. No 172 janvier-mars 2009 : 9-14.

Chemain, Arlette. "L'écriture de Calixthe Beyala: Provocation ou révolte généreuse. » In Notre librairie no 99 octobredécembre, 1989. 162-163.

Chevrier. Jacques. «Calixthe Beyala : quand la littérature féminine africaine devient féministe. » Notre librairie, No 146 octobre-décembre 2001. 22-24.

De Beauvoir, Simone. Le deuxième sexe. I et II. Paris: Gallimard, 1976 (1949).

Emecheta, Buchi. The Joys of Motherhood. Oxford. Heinemann Educational Publishers, 1994.

---. The Bride Price. Oxford: Heinemann Educational Publishers, 1976.

---. Second-Class Citizen. Glasgow: Fontana Books, 1977.

---. The Slave Girl. Glasgow: Fontana Books, 1979.

Figes, Eva. Days. Newcastle Upon Tyne: Faber and Faber Ltd. 1974. 
---. Patriarchal Attitudes. London: Virago Press, 1978.

Gallimore, Rangira B. " De l'aliénation à la réappropriation: Afrique noire Francophone.» Paris: Notre librairie. No 117 Avril-Juin 1994 : 54-60.

Husti-Laboye, Carmen. «Postures féminines dans l'œuvre de Calixthe Beyala. » Présence Francophone. No 75, 2010 : 931.

Iloh, Obiajulum Ngozi. «Femme-Sujet, Femme-Objet: La perception de la femme chez Buchi Emecheta et Henri Lopès. » Thèse inédite, Dept of Foreign Languages, University of Benin, 2009.

Jaccard, Anny-Claire. «Le temps au féminin. » Paris: Notre Librairie. No 117 avril-juin, 1994 : 72-79.

---«Des textes novateurs : la littérature féminine»Paris : Notre librairie. No 99 octobre-décembre 1989: 155-161.

Kom, Ambroise. «L'univers Zombifie de Calixthe Beyala. » Paris :

Notre Librairie. No 125 janvier-mars, 1996 : 64-71.

Nfah-Abbenyi, J.M. Gender in African Women's Writing: Identity, Sexuality, and Difference. Bloomington and Indianapolis: Indiana University Press, 1997.

Onyeoziri, Gloria Nne. "Écriture et oralité dans l'œuvre de Calixthe Beyala. » Présence Francophone. No 75, 2010 : 97-119.

Oyono, Mbia Guillaume. Trois Prétendants un mari. Yaounde: Éditions CLE, 1964.

Sabbah, Hélène. Le commentaire composé. Paris: Hatier, 1993.

Sander, Helke. The Three Ms K. London: The Serpent's Tail, 1987. Schwarzer, Alice. Simone de Beauvoir uujourd'hui: six entretiens.

Traduction française. Paris: Mercure de France, 1984. 
Shakespeare, William. The Taming of the Shrew, qtd in Simone de Beauvoir, The Second Sex. London: David Campbell Publishers Ltd., 1993.

Siwoku-Awi, O.F. «Un labyrinthe sentimental: étude de l'inconscient féminin dans l'œuvre de Calixthe Beyala » In Neohelicon 32 (2005) 1: 241-256.

--- « Tout comme adulte : l'enfant dans le système patriarcal du roman de Calixthe Beyala, Tu t'appelleras Tanga. » Revue nigériane d'etudes françaises. Vol 1, No9, Mars 2007 : 108-130.

--- «Le motif mère-fille dans l'œuvre de Calixthe Beyala: une castration symbolique.» Chuka Chukwube \& Victor Ariole. (eds.) Readings in humanities in honour of Segun Odunuga. Lagos: Fragrance Publishers, 2009 : 237-262.

--- «Une explication psychologique de l'œuvre de Calixthe Beyala, C'est le soleil qui m'a brûlée. »Ogun Journal of Foreign Studies. 2009/2010 No 3: 71-91.

Siwoku-Awi, Tayo. «Woman-Whore-Girl (Child): A Psychorealist Perspective of the Works of Calixthe Beyala. » Sam Ade Ojo (ed.) Feminism in Francophone African Literature. Ibadan: Signal Educational Services, 2003: 186-207. 